1991). Prevalence of migraine diagnosis by IHS criteria also favored Caucasian over African-American children, $61 \%$ to $35 \%$, in a more recent study. Minority children were less likely to present with vomiting, lateralized pain, or food as a precipitant of headache. (Ped Neur Briefs Nov 1993). The IHS criteria should be modified to increase their sensitivity to children and adolescents and also to racial differences in symptomatology. (Progress in Pediatric Neurology II, Chicago, PNB Publ, 1994).

\title{
CLUSTER HEADACHES RELIEVED BY INDOMETHACIN
}

The clinical features and treatment of cluster headaches are reported in two patients, a boy aged 8 and a girl 10 years, evaluated at the Department of Neurology, University of North Carolina at Chapel Hill. Indomethacin $25 \mathrm{mg}$ bid produced immediate and complete relief. The headaches were sharp, unilateral, localized to the temporal region, and associated with lacrimation, nasal congestion, and photophobia. They occurred several times a day for 3 to 4 weeks, sometimes early morning, and lasted 10 minutes to 1 to 2 hours. Clusters were followed by a 2- to 3-week headache-free period. Trials of propanolol, amitryptyline, and biofeedback were unsuccessful. (D'Cruz OF. Cluster headaches in childhood. Clin Pediatr April 1994;33:241-242). (Respond: Dr D'Cruz, Univ of N Carolina, Burnett-Womack Bldg, CB \#7025, Chapel Hill, NC 27599).

COMMENT. Cluster headaches are rare in children and are frequently atypical. Although remarkably effective in the two patients reported, indomethacin toxicity may limit chronic usage. Dietary factors in etiology might be considered.

\section{MOOD AND COGNITIVE DISORDERS}

\section{DYSTHYMIA AND MAJOR DEPRESSIVE DISORDERS COMPARED}

Clinical presentation, course, and outcome of childhood-onset dysthymic disorder (DD) in 55 school-age referrals were compared with a group of 60 youngsters whose first affective episode was major depressive disorder (MDD) in a prospective 3- to 12-year study at Psychiatric Departments of the University of Pittsburgh, Western Psychiatric Institute, University of California at San Diego, and Harvard Medical School. Dysthymic disorder was associated with earlier age at onset than MDD, similarly frequent symptoms of feeling unloved, friendless, irritability, anger, and self-deprecation, but relatively low rates of anhedonia (5\% cf 70\%), guilt (13\% cf 30\%), social withdrawal ( $8 \%$ cf $50 \%$ ), impaired concentration (40\% of $67 \%$ ), loss of appetite (5\% cf $47 \%$ ), insomnia (22\% cf $62 \%$ ), somatic complaints (36\% cf $67 \%$ ) and fatigue (22\% cf $64 \%$ ). Risk of affective disorders, including first-episode MDD $(76 \%)$ and bipolar disorder (13\%), was greater among dysthymic patients. After the first episode of MDD complicating DD, the clinical course of DD was similar to MDD in rates of recurrent major depression and bipolar disorder. In dysthymic children with subsequent MDD, the first episode of MDD is the "gateway" to recurrent affective illness. (Kovacs M et al. Childhood-onset dysthymic disorder. Clinical features and prospective naturalistic outcome. Arch Gen Psychiatry May 1994;51:365-374). (Reprints: Dr Kovacs, Western Psychiatric Institute and Clinic, 3811 O'Hara St, Pittsburgh, PA 15213). 
COMMENT. Pediatric neurologists are frequently faced with the differentiation of organic and psychiatric causes for behavioral and mood disorders and somatic complaints. The recognition of early onset childhood dysthymic disorder should permit prompt referral to colleagues specialized in child psychology and psychiatry. Early diagnosis and treatment of dysthymia may prevent the occurrence of major depressive disorders.

The distinctions between major depression without dysthymia, dysthymia without major depression, and double depression in 62 child psychiatry inpatients were evaluated at the Department of Psychology, State University of New York at Stony Brook. Externalizing disorders (oppositional defiant and conduct disorders) were present more often in the dysthymic group compared to the major depression and double depression groups, whereas major depression and double depression groups revealed higher rates of depressive symptoms. In contrast to the Pittsburgh report, this study found social functioning to be least impaired in children with major depression. (Ferro $\mathrm{T}$ et al. Depressive disorders: distinctions in children. I Am Acad Child Adolesc Psychiatry June 1994;33:664-670).

The role of the Children's Depression Rating Scale-Revised in assessing depression in children with sickle-cell anemia was evaluated at the University of South Alabama Children's Medical Center, Mobile, AL. Excessive fatigue and physical complaints contributed to a high false-positive rate of depression on the standardized screening test, whereas the actual prevalence of depression in these children based on clinical interviews by a child psychiatrist was not increased. (Yang Y-M et al. Depression in children and adolescents with sickle-cell disease. Arch Pediatr Adolesc Med May 1994;148:457-460).

\section{POSTIRRADIATION COGNITIVE AND CNS DISABILITIES}

The neurological, neuropsychological, and educational outcome in 14 children who received a second course of cranial radiotherapy or total body irradiation for relapsing lymphoblastic leukemia is reported from the Neurosciences Unit and Department of Haematology, Institute of Child Health, University of London. Nine (64\%) had postirradiation somnolence syndrome characterized by lethargy, irritability, nausea, and vomiting. All patients had mild neurological deficits, including hyperreflexia, incoordination, dyspraxia, and hand muscle weakness. All were growth hormone deficient. MRI showed ventricular enlargements. Verbal comprehension and tests of attention and memory were impaired, girls showing greater impairments than boys. Of 9 children still at school, only 2 performed at age appropriate levels in reading, spelling, and math, and social outcome was poor. (Christie D et al. Neuropsychological and neurological outcome after relapse of lymphoblastic leukaemia. Arch Dis Child April 1994;70:275-280). (Respond: Dr D Christie, Neurosciences Unit, The Wolfson Centre, Mecklenburgh Square, London WC1N 2AP, UK).

COMMENT. After survival of the rigors of two and three year protocols of chemotherapy for lymphoblastic leukemia, children who relapse after initial remission now have to face the prospect of postirradiation cognitive and neurological deficits. The morbidity asssociated with cranial radiotherapy has been recognized for some time, and the results 